LAWRENCE LIVERMORE NATIONAL LABORATORY

\title{
Computational parametric study of a Richtmyer-Meshkov instability for an inclined interface
}

McFarland, Jacob A., Ranjan, Devesh and Greenough, Jeffrey $A$.

April 4, 2011

Submitted for publication in Physical Review E. 


\section{Disclaimer}

This document was prepared as an account of work sponsored by an agency of the United States government. Neither the United States government nor Lawrence Livermore National Security, LLC, nor any of their employees makes any warranty, expressed or implied, or assumes any legal liability or responsibility for the accuracy, completeness, or usefulness of any information, apparatus, product, or process disclosed, or represents that its use would not infringe privately owned rights. Reference herein to any specific commercial product, process, or service by trade name, trademark, manufacturer, or otherwise does not necessarily constitute or imply its endorsement, recommendation, or favoring by the United States government or Lawrence Livermore National Security, LLC. The views and opinions of authors expressed herein do not necessarily state or reflect those of the United States government or Lawrence Livermore National Security, LLC, and shall not be used for advertising or product endorsement purposes. 


\title{
Computational parametric study of a Richtmyer-Meshkov instability for an inclined interface
}

\author{
Authors \\ Jacob A. McFarland \\ Texas A\&M University Department of Mechanical Engineering, 3123 TAMU College Station, TX 77843-3123 \\ Devesh Ranjan* \\ Texas A\&M University Department of Mechanical Engineering, 3123 TAMU College Station, TX 77843-3123 \\ 979-845-3580 (Tel) \\ 979-845-3081(Fax) \\ * Corresponding Author: dranjan@,tamu.edu \\ Jeffrey A. Greenough \\ Lawrence Livermore National Laboratory, 7000 East Ave. PO Box 808, L-95 Livermore, CA 94550
}

\section{Receipt Date}

February 08, 2011

\begin{abstract}
A computational study of the Richtmyer-Meshkov instability for an inclined interface is presented. The study covers experiments to be performed in the Texas A\&M inclined shock tube facility. Incident Mach numbers from 1.2 to 2.5, inclination angles from 30 to 60 degrees, and gas pair Atwood numbers of $\sim 0.67$ and $\sim 0.95$ are used in this parametric study containing 15 unique combinations of these parameters. Qualitative results are examined through a time series of density plots for multiple combinations of the parameters listed above, and qualitative effects of each of the parameters is discussed. The interface mixing width growth rate is determined for various combinations of the above parameters. A new model for the mixing width growth rate is proposed using the interface geometry and wave velocities calculated using 1D gas dynamic equations. This model uses the transmitted wave velocity for the characteristic velocity and an initial offset time based on the travel time of strong reflected waves. The new model is compared to the Richtmyer Impulsive model and shown to better predict the initial linear mixing width growth rate.
\end{abstract}




\section{INTRODUCTION}

The Richtmyer-Meshkov (RM) instability [1,2] is a hydrodynamic instability occurring due to the misalignment of the pressure and the density gradients. This misalignment generates vorticity through the barolclinic term in the vorticity equation shown below in equation 1, where $\omega$ is the vorticity, D $\omega / \mathrm{DT}$ is the substantial derivative of $\omega, u$ is the velocity, $v$ is the kinematic viscosity, $\rho$ is the density, and $p$ is the pressure.

$$
\frac{D \omega}{D t}=\omega \cdot \nabla u+v \nabla^{2} \omega+\left[\frac{1}{\rho^{2}} \nabla \rho \times \nabla p\right]_{\text {Baroclinic term }}
$$

In the case of the RM instability the pressure gradient is created by an impulsive acceleration of a perturbed fluid interface. The vorticity deposited by this impulsive acceleration will cause the fluid interface to stretch and result in the mixing of the two fluids. The amount of the vorticity deposited will depend on the strength of the pressure and the density gradients. The Atwood number, equation 2 below, is defined as a ratio of densities $\left(\rho_{\mathrm{h}}\right.$ the higher density fluid and $\rho_{1}$ the lower density fluid) at a fluid interface which can be used to describe the effects of the density gradient.

$$
A=\frac{\rho_{h}-\rho_{l}}{\rho_{h}+\rho_{l}}
$$

The RM instability is an important phenomenon to inertial confinement fusion where its occurrence causes the fuel target to mix with inert material and reduces the fuel compression achieved. This reduction in compression greatly reduces the yield of the fuel target. The RM instability is also important in stellar supernova where shock wave interaction with density discontinuities is an important feature. Accurate modeling of the RM instability is also important in supersonic combustion where understanding the mixing of fuel and air is crucial to improving performance. A summary of the RMI instability and its applications is presented in the review article by Brouillette [3]. Another review of the RM instability focusing on the coherent structures formed in flowfield is presented by Zabusky [4].

Various methods for creating the RM instability for experiments have been developed and employed. A brief description of these methods is necessary for perspective on the method discussed in this paper. One method to generate the impulsive acceleration is to use shockwave. Facilities using this method employ a shock tube to generate a shock wave which interacts with a fluid interface containing a density perturbation. An oscillation in the fluid interface can create a sinusoidal perturbation as done by Krivets et al. [5] and Motl et al. [6]. Another interface perturbation method employed in shock tubes is to use a bubble of lighter or heavier gas as used by Ranjan et al. [7]. A third method is to employ a shaped falling gas curtain as described by Prestridge et al. [8]. Finally, alternative methods to generate the impulsive acceleration have been developed as well. One of these methods is to use a sled device which has an impulsive acceleration created by dropping the test section onto a spring at the base of the sled track as described by Chapman and Jacobs [9].

For the study described in this paper the RM instability will be generated by passing a shockwave through a fluid interface. A simple perturbation in the fluid interface can be created by aligning the shockwave at an angle with respect to the density gradient, supported by gravity. The result is an inclined fluid interface with respect to the direction of the impulsive acceleration. This is the method that will be employed in the Texas A\&M University (TAMU) shock tube facility (Figure 1), currently under construction, to study the RM instability. 


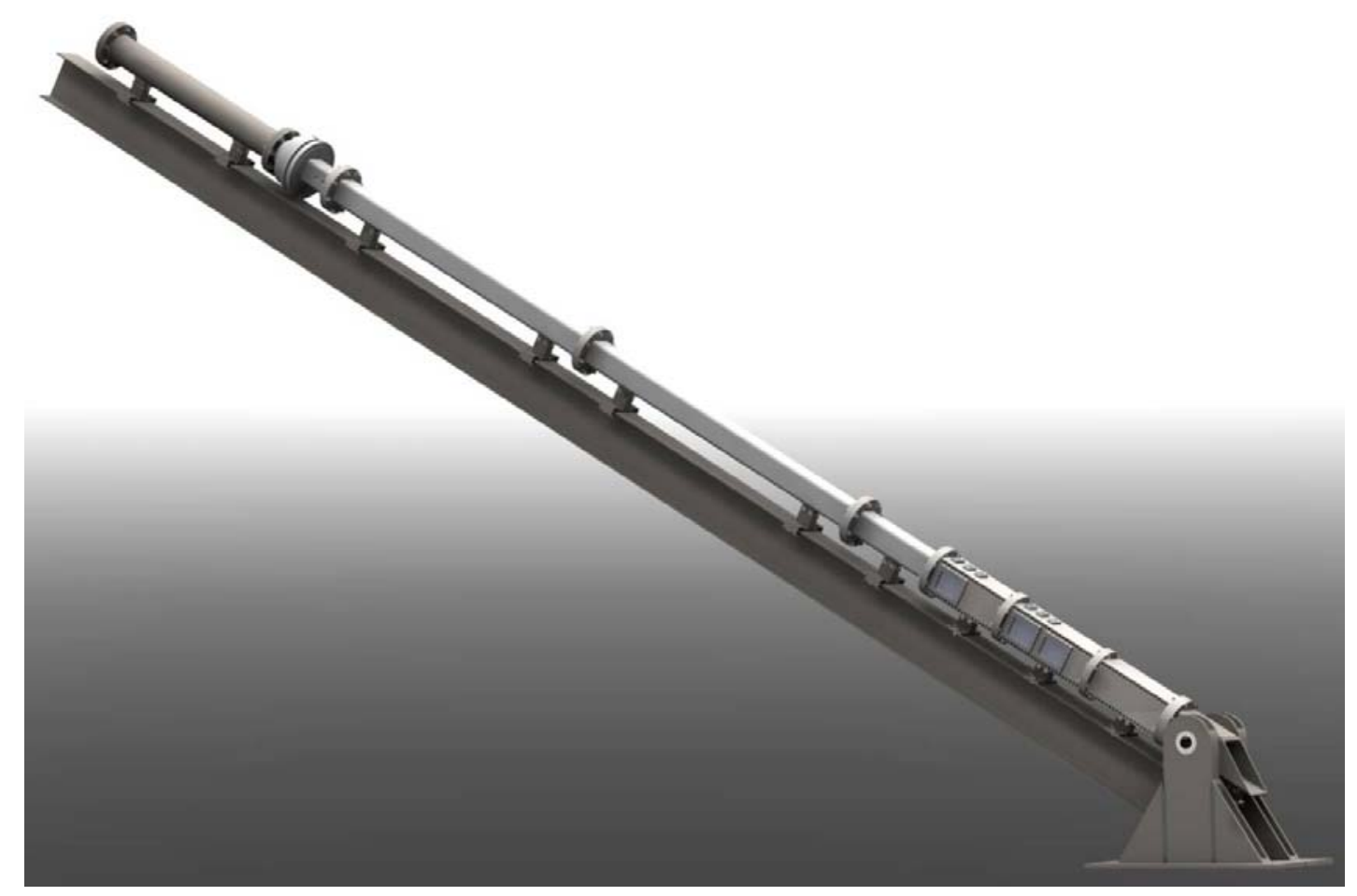

FIG. 1: Texas A\&M Shock tube Facility Currently Under Construction

The simulations presented in the paper were performed in support of the TAMU shock tube facility design, and so the parameter space was limited to the capabilities of this facility. The TAMU shock tube facility will be capable of providing incident shockwave Mach numbers up to 2.5 in atmospheric pressure air, and will be capable of inclination from 0 to 90 degrees. The overall length of the shock tube will be approximately $9 \mathrm{~m}$. The test section will be constructed with a modular design that will allow the interface to be visible at wide range of times with interface visibility up to $1.5 \mathrm{~m}$ downstream of its preshock position. Simultaneous measurements of density and velocity fields will be made using planar laser induced fluorescence (PLIF) and particle image velocimetry (PIV) systems.

\section{COMPUTATIONAL SETUP}

The computation study was performed at Lawrence Livermore National Laboratory using the ARES code developed there. ARES is a staggered mesh Arbitrary Lagrange Eulerian (ALE) hydrodynamics code developed at Lawrence Livermore National Laboratory. The Lagrange time advancement is second-order predictor-corrector and uses the Gauss Divergence theorem to give the discrete finite difference equations [10]. All numerical differences are fully second order in space. Velocities are defined at mesh nodes and density and internal energy are defined at the zone centers using a piecewise constant profile. Artificial viscosity is used to suppress spurious oscillations [11] following the remap phase of the calculation, where the Lagrangian solution is remapped back to a non-Lagrangian mesh, and is fully second order. The original method is given in by Sharp and Baron [12].

The boundary layer simulations use a simple constant viscosity model for constructing the viscous stress. The boundary conditions in these simulations are imposed as solid no-slip, insulated walls. The no-slip wall boundary condition does generate a boundary layer, but it is admitted that it is very far from resolved, but accurate resolution is irrelevant to the current study. The ARES code includes an adaptive mesh refinement (AMR) capability that allows base resolution to be increased by a factor of three for each level of refinement. The 
refinement is performed on areas that exceed an error tolerance in the computation of a second undivided difference of density. The AMR approach used in ARES follows that of Berger and Oliger [13] and Berger and Colella [14]. Time advancement is not recursive though and the coarser levels are slaved to run at the finest level time step.

A 2-D model was used for all simulations presented here. Viscosity was included using a model based on the Sutherland relation shown in equation 3 below, where $\mu$ is the dynamic viscosity, $T$ is the temperature, and $F_{1}$ and $F_{2}$ are density and pressure correction factors that were set to one for the model used. Coefficients for the gases used are presented in table I below. The inclusion of the viscosity is discussed in the appendix. The present simulations did not include species diffusion, or stratification which will be included in future work.

$$
\mu=\left[\left(v_{c}+v_{a} \cdot \frac{T^{v_{n}}}{v_{b}+T}\right) F_{1}\right] F_{2}
$$

Table I: Summary of coefficients used in Sutherland relation.

\begin{tabular}{|l|l|l|l|l|}
\hline Gas & $\begin{array}{l}\mathrm{V}_{\mathrm{a}} \\
\left(\mathrm{g} / \mathrm{cm}-\mathrm{us}-\mathrm{K}^{\wedge} .5\right)\end{array}$ & $\begin{array}{l}\mathrm{V}_{\mathrm{b}} \\
(\mathrm{K})\end{array}$ & $\begin{array}{l}\mathrm{V}_{\mathrm{c}} \\
(\mathrm{g} /(\mathrm{cm}-\mathrm{us})\end{array}$ & $\mathrm{V}_{\mathrm{n}}$ \\
\hline Air & $1.45 \mathrm{E}-11$ & 110.4 & 0 & 1.5 \\
\hline SF6 & $1.59 \mathrm{E}-11$ & 243.8 & 0 & 1.5 \\
\hline
\end{tabular}

Preliminary simulations were run with a computational domain that included the entire shock tube. To reduce computational times for the parametric study the domain was decreased to $2.5 \mathrm{~m}$. The shock was initialized $1 \mathrm{~cm}$ ahead of the upstream most end of the interface, which varied in position for different inclination angles. The downstream and sidewall boundary conditions were reflecting walls with no slip conditions. The downstream wall was placed at similar position to that of a configuration of the TAMU shock tube to allow for more accurate simulations of reshock. A section of the computational domain for the initial conditions is shown below in figure 2 where the upstream and downstream boundary conditions are at 0 and $250 \mathrm{~cm}$ respectively.

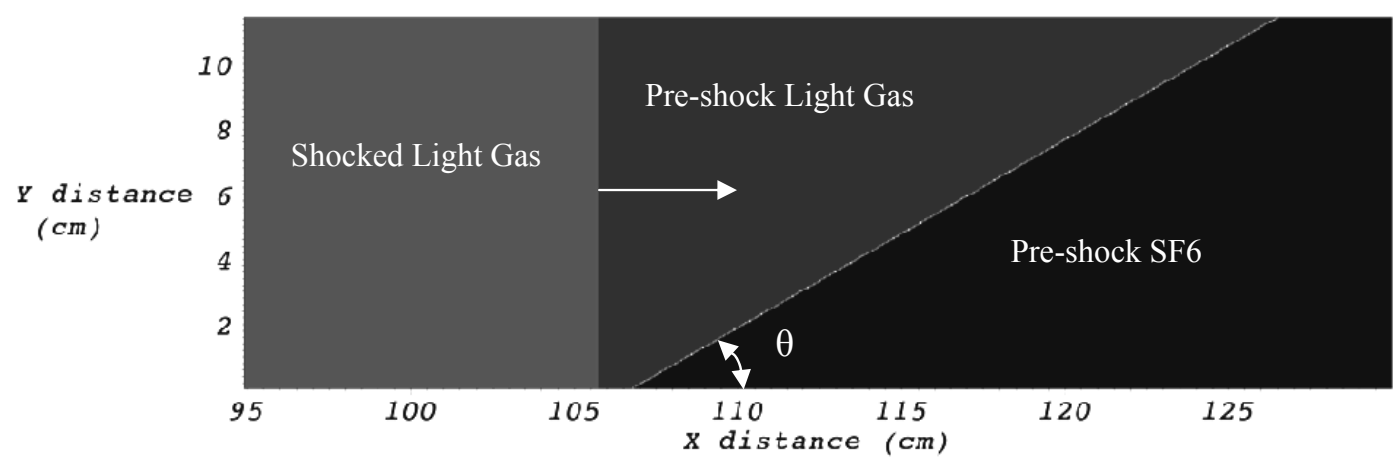

FIG. 2: Density plot of the initial conditions for an interface inclination angle of 30 degrees.

The upstream boundary consisted of a reflecting wall with a source term added which supplied the necessary inflow to support the shockwave. This boundary condition was not ideal as interface reflected shockwaves traveling upstream were reflected back towards the interface as expansion waves. The intersection of the reflected expansion waves with the shocked interface limited the time over which the simulation could be used to model experiments in the TAMU shock tube facility. 
The parametric study was run for 3 different inclination angles; 30, 45, and 60degrees. Two different gas pairs were used at each angle, air-SF6 ( $\left.A_{t} \sim 0.67\right)$, and helium-SF6 ( $\left.A_{t} \sim .95\right)$. The air-SF6 interface was run at three different Mach numbers; 1.5, 2.0, and 2.5. The heliumSF6 interface was run at Mach 1.2 and 1.5. The study parameters are summarized below in Table II. Due to the large number of simulations that were required to cover the various parameters the AMR capability was particularly useful. The AMR capability reduced the computational time and allowed for higher resolution studies with limited resources.

Table II: List of study simulations

\begin{tabular}{|l|l|l|l|}
\hline Case & $\mathbf{M}$ & $\boldsymbol{\theta}$ & Gas Pair \\
\hline 1 & 1.5 & 30 & Air-SF6 \\
\hline 2 & 1.5 & 45 & Air-SF6 \\
\hline 3 & 1.5 & 60 & Air-SF6 \\
\hline 4 & 2.0 & 30 & Air-SF6 \\
\hline 5 & 2.0 & 45 & Air-SF6 \\
\hline 6 & 2.0 & 60 & Air-SF6 \\
\hline 7 & 2.5 & 30 & Air-SF6 \\
\hline 8 & 2.5 & 45 & Air-SF6 \\
\hline 9 & 2.5 & 60 & Air-SF6 \\
\hline 10 & 1.2 & 30 & He-SF6 \\
\hline 11 & 1.2 & 45 & He-SF6 \\
\hline 12 & 1.2 & 60 & He-SF6 \\
\hline 13 & 1.5 & 30 & He-SF6 \\
\hline 14 & 1.5 & 45 & He-SF6 \\
\hline 15 & 1.5 & 60 & He-SF6 \\
\hline
\end{tabular}

\section{QUALITATIVE FLOW FIELD DESCRIPTION}

A time series of density plots for four different parameter sets showing the effect of changing each of the 3 parameters is presented below in Figure 3. The exemplar case (Fig. 3 set $\mathrm{B}$, Case $1, \mathrm{M}_{\mathrm{i}}=1.5, \theta=30^{\circ}, \mathrm{A} \sim 0.67$ ) is displayed along with three other cases differing in incident shock Mach number (Fig. 3 set $A$, Case 7, $\mathrm{M}_{\mathrm{i}}=2.5, \theta=30^{\circ}, \mathrm{A} \sim 0.67$ ), Atwood number (Fig. 3 set $\mathrm{C}$, case $13, \mathrm{M}_{\mathrm{i}}=1.5, \theta=30^{\circ}, \mathrm{A} \sim 0.95$ ), and interface inclination angle (Fig. 3 set $\mathrm{D}$, case $3, \mathrm{M}_{\mathrm{i}}=1.5, \theta=60^{\circ}, \mathrm{A} \sim 0.67$ ) respectively. All three cases evolve a region of SF6 with low mixing that follows the transmitted shock front. After some time the mixing region separates from this region of SF6, and the transmitted shock and the interface separating the post shock light gas and this region of SF6 both become planar. This region of SF6 will be referred to as the slug in the following discussion due to its rectangular shape.

The exemplar case (Fig. 3, set B) will be used as the basis for comparison to the other three cases displayed in Fig. 3. This case is characterized by a large mixing and slug region. A strong lambda shock (Fig.3, B1) is formed which travels up the slug region until impacting and reflecting off the upper wall. This reflection creates many more secondary compressible effects of moderate strength that resonate within the slug (Fig. 3, B4). A weak Winkler vortex structure [15] is formed within the slug (Fig. 3, B3). As the mixing region continues to develop in time it separates into two large secondary structures of SF6 (Fig. 3, B5) which persist and exhibit a lower degree of mixing. 


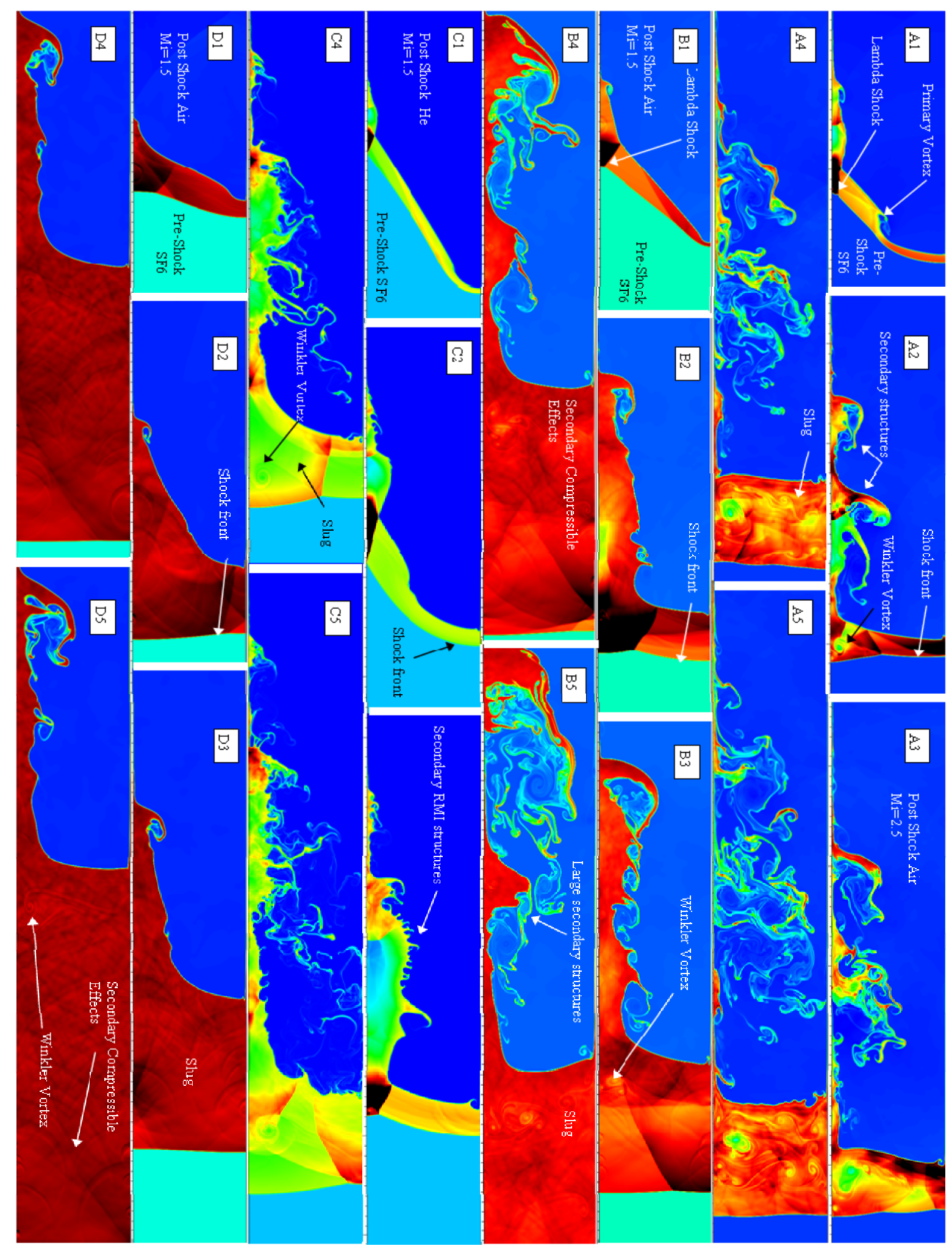

FIG. 3: Time series plot of density for three different parameter sets. Figures A1-A5, Case 7, at times 0.5, 1.0, 1.5, 2.0, and $2.5 \mathrm{~ms}$ respectively. Figures B1-B5, Case 1, at times 0.5, 1.5, 2.5, 4.0, and 5.5 ms. Figures C1-C5, Case 13, at times 0.25, 0.5, 1.0, 1.5, and 2.0 ms. Figures D1-D5, Case 3, at times 0.5, 1.5, 2.5, 4.0, and $5.5 \mathrm{~ms}$. 
The effects of incident shock wave Mach number can be examined by comparing the exemplar case (Fig. 3, set B) with case 7 (Fig. 3, set A). These two cases differ only in the strength of the incident shock. In case 7 the stronger incident shock leads to a higher degree of separation between the mixing region and the slug. In case 7 the transmitted, lower wall reflected shock is flattened with the interface by the interface reflected, upper wall reflected shock and then separated by the primary vortex created at the upper wall which has traveled down the interface. This results in the early destruction of the lambda shock at the lower wall allowing the primary transmitted shock to replanarize quickly. The destruction of the lambda shock and the limited width of the slug region suppress the secondary compressible effects within the slug seen in the exemplar case. A strong Winkler vortex structure is also created within the slug after the lambda shock is destroyed (Fig. 3, A2). The Winkler vortex is joined by other smaller vortical structures at later times (Fig. 3, A5)

The effect of the interface Atwood number is shown by comparing the exemplar case (Fig. 3, set B) with case 13 (Fig. 3, Set C). Case 13 differs only from the exemplar case in Atwood number, but the incident shock strength of Mach 1.5 in helium is more like the shock strength of case 7, Mach 2.5 in air. In case 13 the higher Atwood number increases the strength of shockwaves reflected from the interface and decreases the strength of refracted waves. This ultimately results in the suppression of the large secondary structures created by the first interface reflected, upper wall reflected, transmitted shock seen in case 7 and to a lesser degree in the exemplar case. In case 13 smaller secondary structures then become visible that are created from a second RM instability. This second RM instability is created by the refraction of a leg of the lambda shock, moving from heavy to light gas, which is reflected from the upper wall and then refracted again through the interface, light to heavy. The strength of the refracted shock is weaker than that of the exemplar case and case 7 but its wall reflection's interaction with a relatively flat helium-SF6 interface makes the secondary structures produced visible.

Interface inclination effects are shown by comparing the exemplar case (Fig. 3, set B) with case 3 (Fig. 3, set D). Case 3 differs only in inclination angle $\left(\theta=60^{\circ}\right)$ from the exemplar case. Case 3 is characterized by slower interface growth and a smaller, less mixed, mixing region due to the decreased inclination angle. This inclination angle provides particularly uniform properties in the post shock SF6, due to the weakness of reflected secondary compressible effects (fig. 3, D5). The lack of strong reflected compressible effects also results in a simpler interface with fewer secondary spikes and limited mixing. A weak Winkler vortex can be seen within the slug.

\section{QUANTITATIVE RESULTS}

\section{A. Previous linear growth rate models}

For this paper the non-dimensional growth rate of the mixing region was examined in the linear region. Previous models have attempted to estimate the mixing width growth rate in the linear region for different kinds of interfaces. Richtmyer [1] first proposed an impulsive growth model for a sinusoidal interface in 1960 based on Taylor's [16] work of 1950. The impulsive growth rate equation is presented below in Equation 4 where $\eta, \mathrm{k},[v], \eta_{0}$, are the interface mixing width, wave number, velocity jump, and preshock initial amplitude respectively.

$$
\dot{\eta}_{0}=k[v] A \eta_{0}
$$

The post shock Atwood number and the post shock interface amplitude can be used to increase the accuracy of Equation 4 in the light to heavy gas shock case. To estimate the post 
shock amplitude Meshkov [2] introduced the relationship shown in Equation 5 below where $w_{\mathrm{i}}$ is the incident shock speed.

$$
\eta_{0}^{\prime}=\eta_{0}\left(1-\frac{|[v]|}{w_{i}}\right)
$$

Various modifications to improve the impulsive model for both light/heavy and heavy/light interfaces have been made by Myer and Blewett [17], and Vandenboomgaerde [18]. A growth reduction factor was proposed based on experiments for a diffuse interface by Motl et al. [6].

While many attempts have been made to model the linear mixing width growth for sinusoidal interfaces, few have been presented for an inclined interface. Work on inclined interfaces has been limited thus far to numerical simulations. Circulation deposition on an inclined gas curtain was examined in simulations by Samtaney and Zabusky [19]. Numerical simulations to study vortex dynamics in an inclined gas curtain were presented by Zhang et al. [20]. The linear growth of arbitrary interface shapes, including a "v" shaped interface equivalent to an inclined interface, is studied in numerical simulations by Mikaelian [21].

\section{B. Mixing width definition}

Before a mixing width growth rate model can be applied and tested the mixing width must be defined. One method to define the mixing width is to use the width for an equivalent perfectly mixed region. This is the procedure is described by Cabot and Cook [22]. Another technique used is to define the mixing width as the region between the 5 and $95 \%$ mole fraction of one of the species. By starting with the 5\% concentration of either species the mixing width is limited to the region occupied by a substantial amount of either species. This eliminates the effects of spurious small mass projectiles that escape the main structure and fluid entrained in the boundary layer. The disadvantage of this method is that mixing width can discontinuously jump in size as a fluid is pulled out away from the current mixing region and accumulates to a level where it passes the 5 or $95 \%$ concentration level (Figure 4). The equivalent mixing width method avoids this problem by integrating over the entire molar concentration levels. The advantage of the 5 to $95 \%$ concentration method is that it provides a length that has a physical representation easily seen in simulation results. For this reason the 5 to $95 \%$ concentration measurement was used to define the mixing width. 


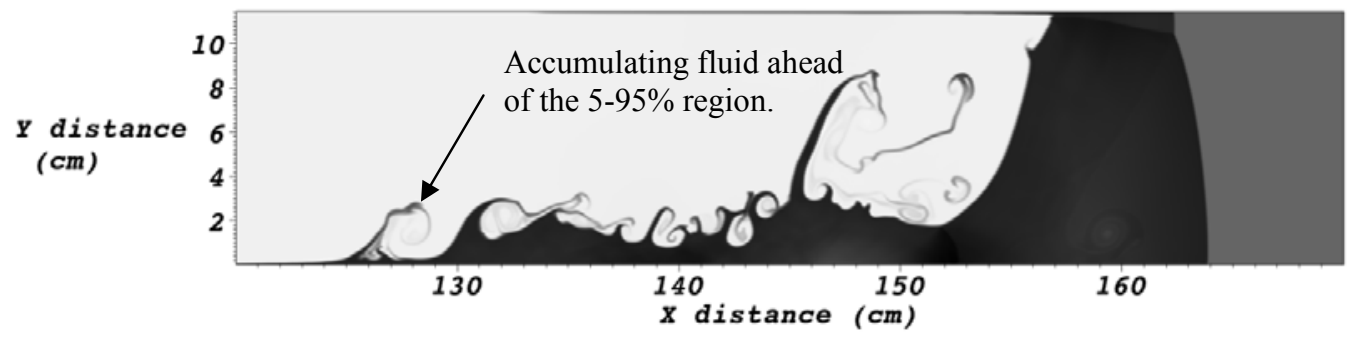

FIG 4: Density Plot Showing Accumulation of Fluid Outside of the Calculated Mixing Width Region (5\%-95\% Species Concentration).

\section{Adaptation of Richtmyer impulsive model}

The Richtmyer impulsive model was first tested to determine its ability to match the simulation data. To use the Richtmyer impulsive model for these simulations some variables had to be redefined in the context of an inclined interface. The initial interface amplitude was defined as the length of the interface measured in the direction of travel of the incident shockwave (Figure 5). The interface wavelength was defined as the length of the interface perpendicular to the direction of travel of the incident shockwave. This distance is the width of the shock tube as well.

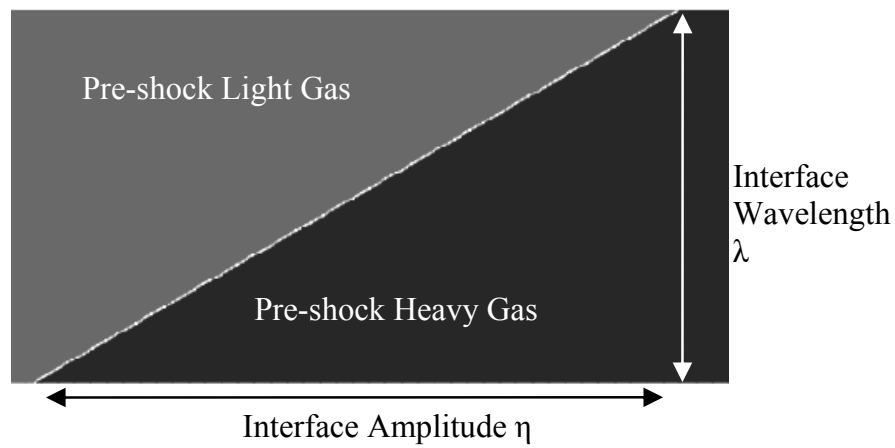

FIG. 5: Interface Amplitude and Wavelength for the Richtmyer Impulsive Model.

The Richtmyer impulsive model was used to non-dimensionalize the results of the simulations. A non-dimensional time and mixing width are defined below in Equations 6 and 7 using the impulsive growth model with the post shock Atwood number and amplitude (Equation 8). The Richtmyer impulsive model predicts the growth of an interface after the incident shockwave has interacted with the entire length of the interface. Because of this the non-dimensional time $(\tau)$ is offset from the simulation time to be zero at the time the incident shockwave has completely traversed the initial amplitude of the interface. This offset time is defined in Equation 9. The choice of this time is important as it predicts the time at which the initial non-linear compression of the interface mixing width ends and linear growth regime begins. The non-dimensional mixing width is offset so that it starts at zero when $\tau$ equals zero (Equation 10). The 1D gas dynamic parameters and wave speeds used in these calculations and calculations in the following section are listed in Table III and Table IV below.

$$
\begin{aligned}
& \tau=k \dot{\eta}_{0}\left(t-t^{*}\right) \\
& \bar{\eta}=k\left(\eta-\eta_{0}^{\prime}\right) \\
& \dot{\eta}_{0}=k \eta_{0}^{\prime} A^{\prime}[v]
\end{aligned}
$$




$$
\begin{aligned}
& t^{*}=(\lambda / \tan (\theta)) / w_{i} \\
& \eta_{0}^{\prime}=\eta\left(t^{*}\right)
\end{aligned}
$$

Table III: 1D gas dynamics values.

\begin{tabular}{|l|r|r|l|l|}
\hline $\begin{array}{l}\text { Initial } \\
\text { Parameters }\end{array}$ & \multicolumn{1}{|l|}{ Gamma } & $\mathrm{R}(\mathrm{j} / \mathrm{kg}-\mathrm{K})$ & $\begin{array}{l}\text { Initial Gas } \\
\text { Temperature } \\
(\mathrm{K})\end{array}$ & $\begin{array}{l}\text { Initial Gas } \\
\text { Pressure }(\mathrm{kPa})\end{array}$ \\
\hline Air & 1.4 & 287 & 300 & 101.3 \\
\hline Helium & 1.667 & 2077 & 300 & 101.3 \\
\hline SF6 & 1.09 & 56.92 & 300 & 101.3 \\
\hline
\end{tabular}

Table IV: 1D gas dynamics calculated wave speeds.

\begin{tabular}{|r|l|l|r|r|r|}
\hline $\begin{array}{l}\text { Incident Mach } \\
\text { Number }\end{array}$ & Light Gas & Heavy Gas & wi (m/s) & wt $(\mathrm{m} / \mathrm{s})$ & wrrt $(\mathrm{m} / \mathrm{s})$ \\
\hline 1.2 & Helium & SF6 & 1223.00 & 192.73 & 156.34 \\
\hline 1.5 & Helium & SF6 & 1528.76 & 282.05 & 177.56 \\
\hline 1.5 & Air & SF6 & 520.75 & 242.76 & 156.56 \\
\hline 2 & Air & SF6 & 694.38 & 353.75 & 167.23 \\
\hline 2.5 & Air & SF6 & 867.97 & 465.55 & 197.17 \\
\hline
\end{tabular}

The non-dimensionalized data from the Richtmyer impulsive model for the Air-SF6 simulations is plotted below in Figure 6. For all mixing width plots the key lists the cases described by the light gas name, the Mach number, excluding the decimal, preceded by the letter M, and inclination angle preceded by the letter A. From Figure 6 it can be seen that the model collapses the simulation results together well based on incident shockwave Mach number. However the data does not collapse well for the three different interface inclination angles. Also initial agreement at $\tau<25$ is not good as compared to later $\tau$. This is partially due to the discontinuities in mixing width measurements discussed in section $\mathrm{B}$, but also due to additional non-linear growth that occurs after the incident shockwave traverses the interface initial amplitude. A sudden decrease in the mixing width at the end of some data sets is due to the onset of reshock from the downstream boundary that was not entirely cropped off of the data set. Figure 7 below shows Helium-SF6 simulation data compared to a sample of the air-SF6 data. This figure illustrates the performance of this model for different Atwood numbers. The agreement is quite poor again, but it can be seen that the Helium-SF6 simulations fall in the same range of non-dimensional growth rates as the air-SF6 simulations. This suggests that the problem in the Richtmyer impulsive model is not its ability to collapse data for different Atwood numbers but its ability to collapse data for different interface inclination angles. 


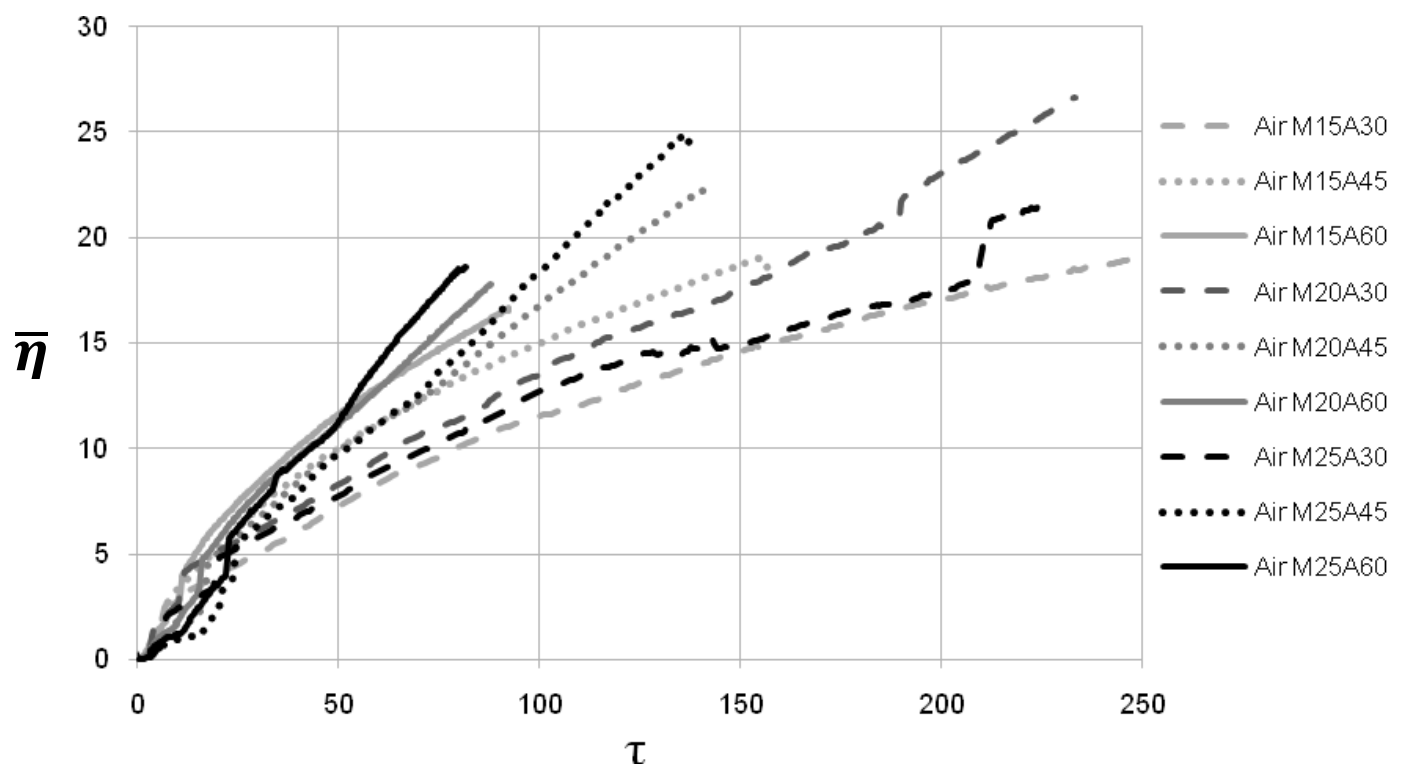

FIG. 6: Non-dimensional mixing width versus time for all air-SF6 parameter sets where $\bar{\eta}$ is the non-dimensional mixing width, and $\tau$ is the non-dimension time.

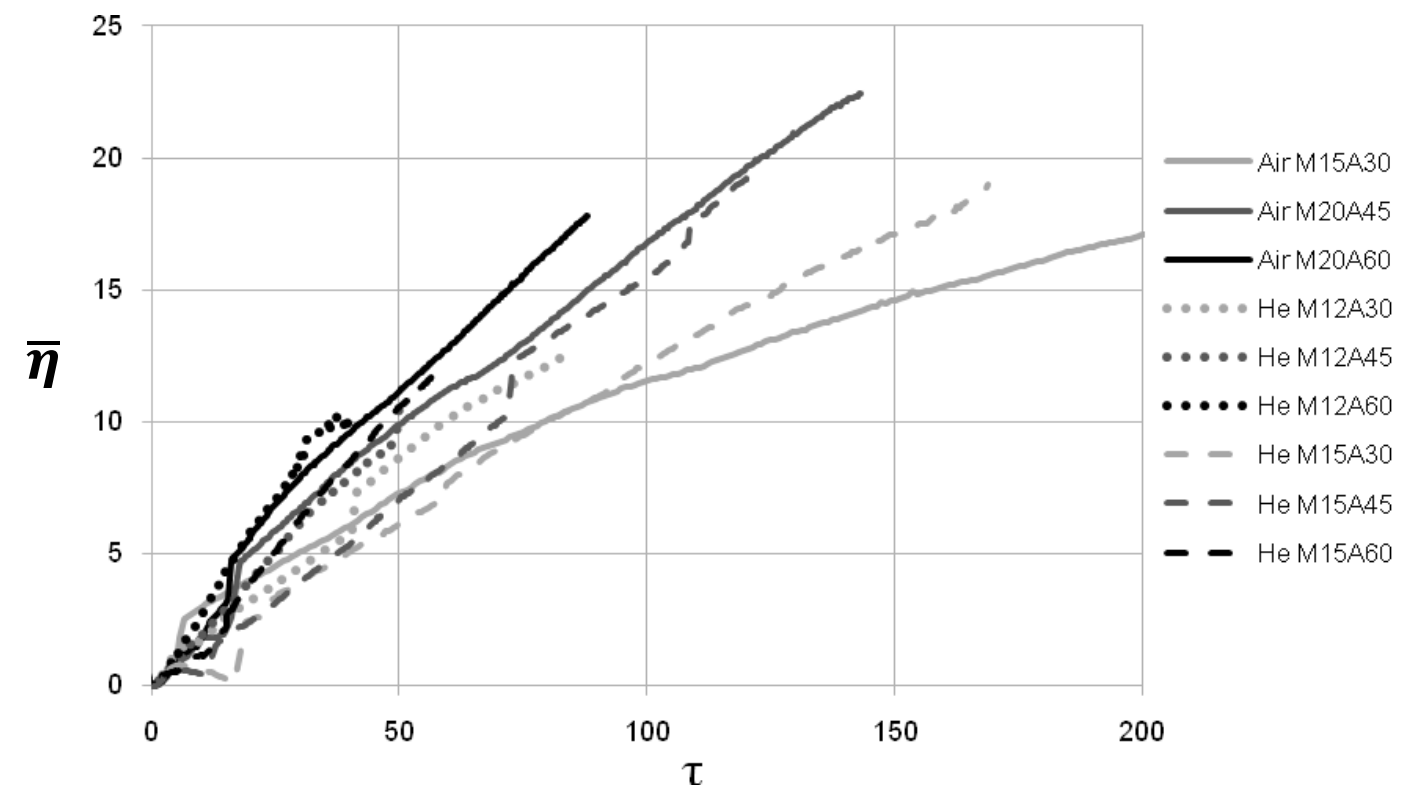

FIG. 7: Non-dimensional mixing width versus time for selected air-SF6 and helium-SF6 parameter sets where $\bar{\eta}$ is the non-dimensional mixing width, and $\tau$ is the nondimension time.

\section{Inclined interface impulsive model}

From the Richtmyer impulsive model it can be seen that for a model to fit the inclined interface simulation data it needs to account for the interface geometry and the initial nonlinear compression of the interface. The strategy for arriving at a new linear growth model 
was to use the basic pattern of the impulsive model but to select parameters appropriate to an inclined interface. The impulsive model consists of an offset time multiplied by a gas pair ratio and divided by characteristic time (equation 11). Since the impulsive model matched different Atwood number cases well the gas pair ratio parameter, Atwood number, was not changed.

$$
\tau=\frac{A\left(t-t^{*}\right)}{t_{\text {char }}}
$$

The characteristic time in the impulsive model was found from a characteristic velocity, the interface jump velocity, and a characteristic length, the wave number squared divided by the post shock interface amplitude. For the new model it was found that the transmitted shockwave speed $\left(w_{\mathrm{t}}\right)$ was a more accurate characteristic velocity. This is because the important secondary compressible effects (interface reflected, wall reflected, interface transmitted $\left(\mathrm{I}_{\mathrm{R}} \mathrm{W}_{\mathrm{R}} \mathrm{I}_{\mathrm{T}}\right)$ shockwave, and subsequent reflections) travel at speeds that scale more closely with the transmitted wave speed. The importance of these secondary compressible effects is explained further with respect to the selection of an offset time later.

The characteristic length was selected by examining the effects of the length of the interface parallel (height) and perpendicular (width) to the incident shockwave velocity. The result was a characteristic length term called the effective $\lambda$ (Equation 12). This effective $\lambda$ can be described as the interface width that would be required for each inclination angle if the interface height were held constant across all inclination angles. The resulting characteristic length is defined as $\lambda^{2} / \eta_{0}$. This is similar to the characteristic length for the Richtmyer impulsive model when modified as described before (section $C$ ) for an inclined interface ( $\left.\lambda^{2} / 4 \pi^{2} \eta_{0}^{\prime}\right)$. The difference is that the adapted Richtmyer impulsive model uses the post shock compressed amplitude and the new model uses the initial interface height. This is because the post shock compressed amplitude is more complicated to predict due to secondary compressible effects, while the initial interface height is simple and produced a more accurate model.

$$
\lambda_{E}=\lambda \cdot \tan (\theta)
$$

Another improvement in the new model was to use a more accurate offset time to better predict the initial non-linear compression time. The Richtmyer impulsive model was offset by the time it took for the incident shockwave to traverse the height of the interface. This accounts for the initial compression of the interface by the incident shockwave but misses the compression effects of the secondary compressible waves. After the incident shockwave is transmitted secondary compression and expansion waves continue to traverse the width of the interface. These waves increase in strength as the interface inclination angle becomes more oblique (low $\theta \mathrm{s}$ ). The strength of these waves diminishes with every interface and boundary interaction that occurs. The first of these secondary effects is shown below in Figure 8 . This wave is denoted as the interface reflected, wall reflected $\left(\mathrm{I}_{R} \mathrm{~W}_{R}\right)$ shockwave. The strength of this shockwave is sufficient to cause a compression of the interface along its width, and to extend the initial non-linear growth of the interface. Subsequent waves will continue to alter the growth rate at a diminishing rate. The time at which this wave has compressed the interface will approximate the end of the initial non-linear growth rate. 


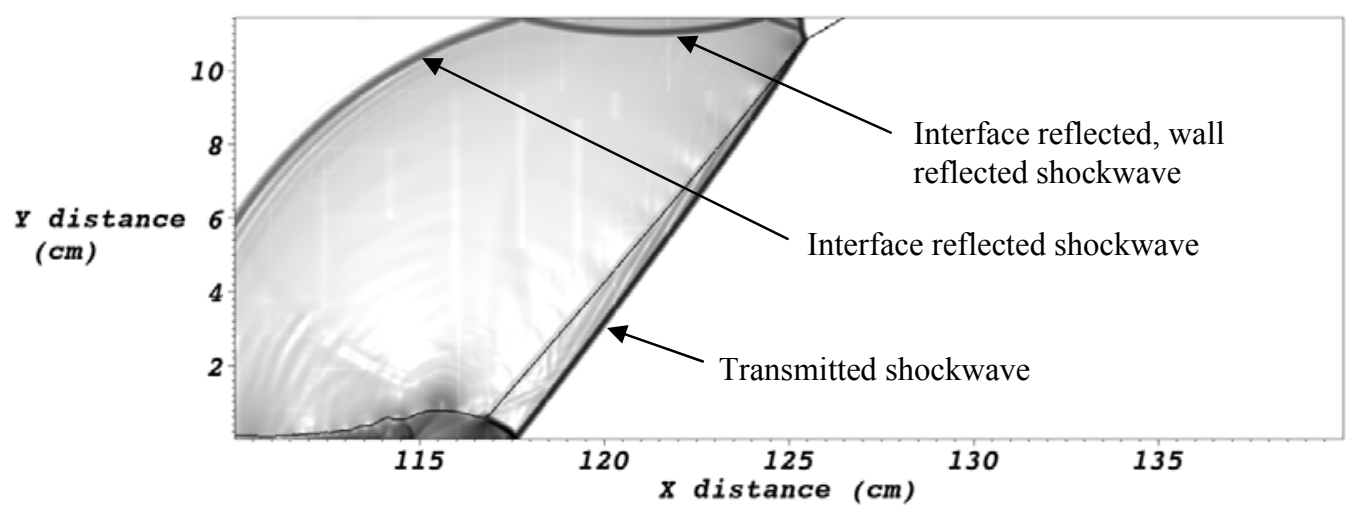

\section{FIG. 8: Gradient of pressure field plot at early time (t $\sim 226 \mathrm{~ms})$.}

To predict the time at which the $I_{R} W_{R}$ shockwave has compressed the interface 1-d gas dynamic equations were used. The initial compression time from the incident shockwave was again calculated using the 1-d incident shock speed and the interface height. At the end of this time it was assumed that part of $\mathrm{I}_{R} \mathrm{~W}_{\mathrm{R}}$ shock transmits through the interface. The transmitted leg of this wave $\left(I_{R} W_{R} I_{T}\right)$ was used to determine the end of the initial nonlinear growth since it had the lower speed. While the 1-d calculated speed of the un-transmitted leg of the wave is higher the wave is limited by the transmitted leg (Figure 9). Using the 1-d approximated speed of the transmitted leg more accurately predicted the time at which the transmitted leg has reached the wall (Figure 10) and the compression of the interface from this wave is complete. The width compression time is then determined from the 1-d velocity of the $\mathrm{I}_{\mathrm{R}} \mathrm{W}_{\mathrm{R}} \mathrm{I}_{\mathrm{T}}$ shockwave $\left(w_{\text {rrt }}\right)$, and the width of the interface. This time is added to the initial compression time to yield the new offset time shown in Equation 13. Calculated 1D wave speeds used in Equation 13 are shown in Table IV of the previous section. Plots of the nondimensional growth rate versus non-dimensional time (Figure 11) as well as qualitative examination of density field plots showed that this offset time was a good predictor of the end of the initial nonlinear compression.

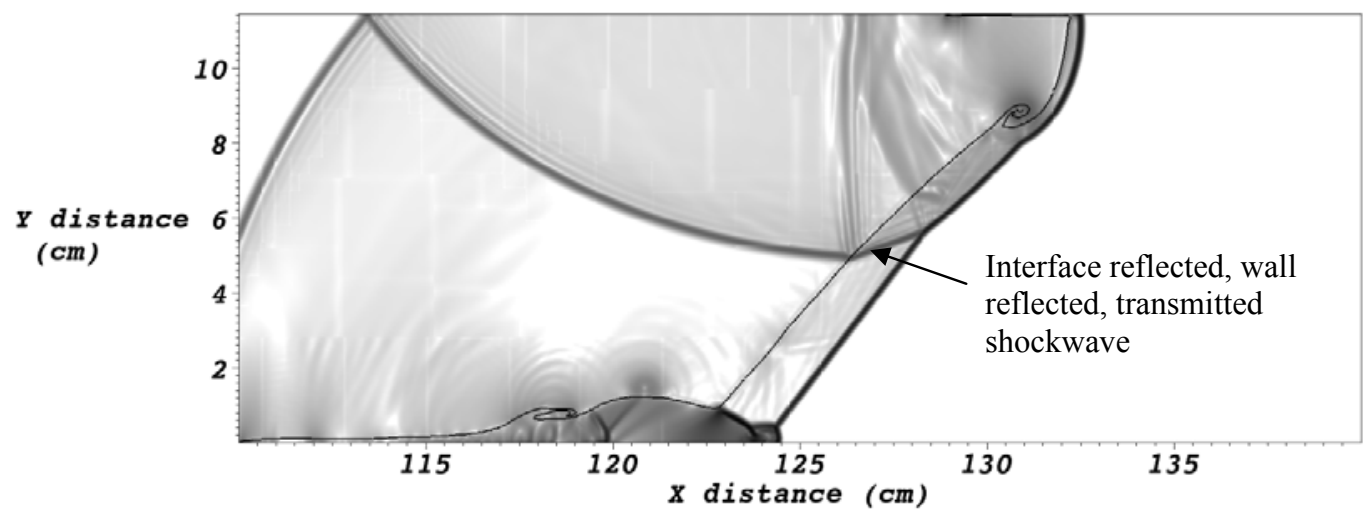

FIG. 9: Gradient of pressure field plot (t $\sim .302 \mathrm{~ms})$. 


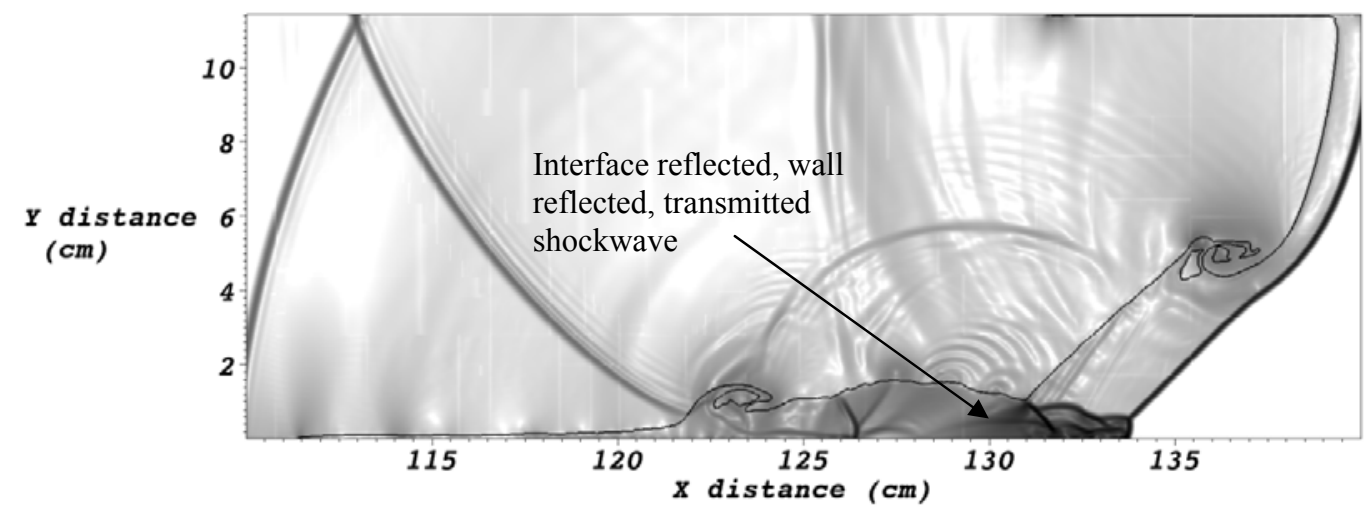

FIG. 10: Gradient of pressure field plot (t .524ms).

$$
t^{*}=\frac{(\lambda / \tan (\theta))}{w_{i}}+\frac{\lambda}{w_{r r t}}
$$

Equations for the new linear growth are shown below in equations 14-16. The calculated 1D transmitted wave speed used in Equation 14 can be found in Table IV of the previous section. The results of the new linear growth model are presented below in Figures 11 and 12. From these figures it can be seen that the initial agreement for the air-SF6 gas pair has improved over the Richtmyer model. The divergence of the different Mach number cases occurs much earlier in the new non-dimensional time. The new non-dimensional time also creates a larger separation in the non-dimensional development rate of the different inclination angle cases. It would appear from Figure 12 that the agreement between heliumSF6 cases is not as good, but the errors in the mixing width algorithm discussed in section B cause jumps in the mixing width that offset the non-dimensional mixing width. If the slopes after these jumps are compared it can be seen that the growth rates are similar for all cases. A different definition of the mixing width or a modification of the current definition could mitigate these jumps, and will be considered in future work.

$$
\begin{aligned}
\tau_{a} & =\frac{w_{t}}{\lambda_{E}}\left(t-t^{*}\right) \\
\bar{\eta}_{a} & =\frac{\left(\eta-\eta^{*}\right)}{\lambda_{E}} \\
\eta^{*} & =\eta\left(t^{*}\right)
\end{aligned}
$$




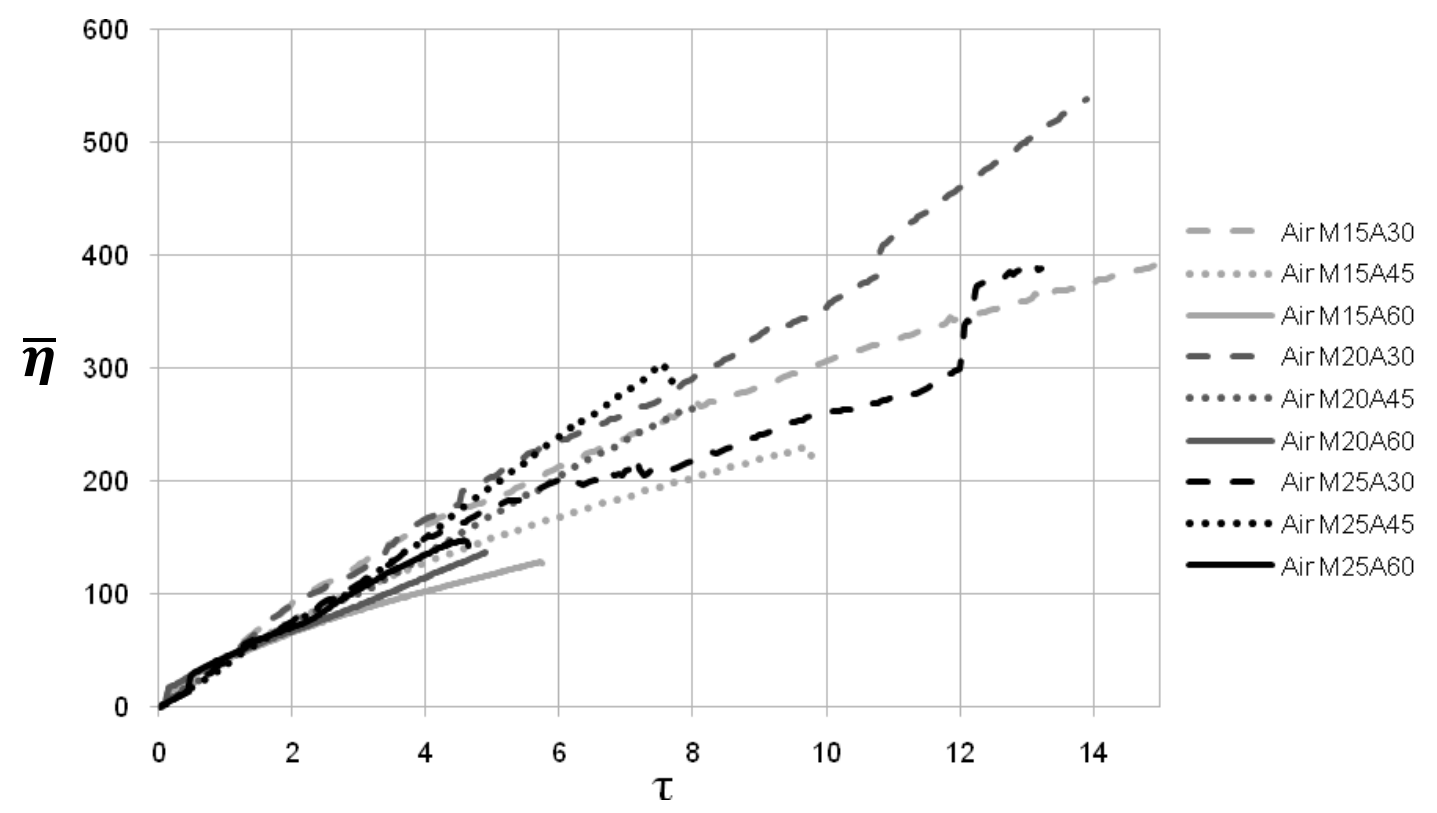

FIG. 11: Non-dimensional mixing width versus time for all air-SF6 parameter sets where $\bar{\eta}$ is the non-dimensional mixing width, and $\tau$ is the non-dimension time defined using the new linear growth model.

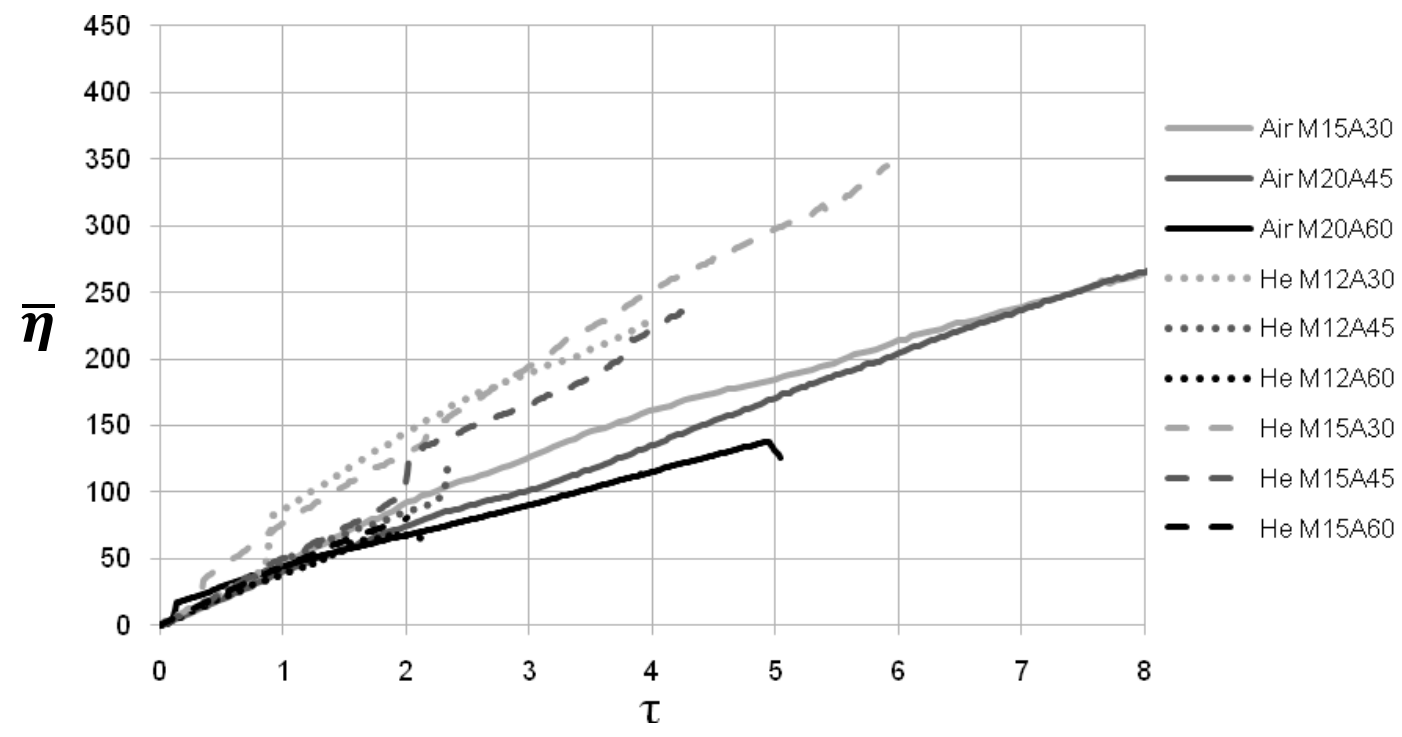

FIG. 12: Non-dimensional mixing width versus time for selected air-SF6 and heliumSF6 parameter sets where $\bar{\eta}$ is the non-dimensional mixing width, and $\tau$ is the nondimension time defined using the new linear growth model.

\section{CONCLUSIONS}

There is much to be explored still for the inclined interface RM instability. This type of interface produces many unique features and challenges. From the qualitative results 
presented in this paper it can be seen that the inclination angle, incident Mach number, and gas pair properties have a strong effect on the development of the interface. The linear growth rate of the mixing width is predicted poorly using the Richtmyer impulsive model adapted for the inclined interface. The Richtmyer impulsive model struggles to collapse the data from different inclination angles. A new linear mixing width model was proposed that collapses the data for all parameters sets well at early times. The new model uses the 1-d wave speeds for the incident shockwave and the interface reflected, wall reflected, transmitted shockwave to predict the end of the initial nonlinear growth. The characteristic velocity for the new model was the 1-d transmitted wave speed instead of the interface jump velocity used in the Richtmyer impulsive model. Future work will seek to define the interface mixing width better and to explore the divergence between the interface angles at later times.

\section{ACKNOWLEDGEMENTS}

Jacob A. McFarland would like to thank Robert Morgan, and Chris Weber for the consultation, advice and support while this research was conducted. Also, to thank Prof. Ricardo Bonazza for his advice and helpful discussion of the work presented in this paper. Additionally this work could not have been performed without the High Energy Density Physics Summer Student Program which provided Jacob A. McFarland with the opportunity

to work with Jeff Greenough and many other highly knowledgeable and helpful employees of LLNL. Finally, this work performed under the auspices of the U.S. Department of Energy by Lawrence Livermore National Laboratory under Contract DE-AC52-07NA27344. 


\section{APPENDIX A: RESOLUTION STUDY}

A resolution study was first performed to determine the maximum resolution that could be achieved while limiting the simulation computer run times to a level that would allow the study to be completed in a two month period. The most computationally time intensive simulation used the following parameters; incident shockwave Mach number $\left(\mathrm{M}_{\mathrm{i}}\right)$ of 2.5 , interface inclination angle of ( $\left.\mathrm{A}_{\text {int }}\right) 30^{\circ}$, and an Atwood number of $\sim 0.67$ (air-SF6 interface). Several simulations were started for this set of parameters with different resolutions to estimate the computation time it would take for the models to run to $4 \mathrm{~ms}$. This time was chosen as the cutoff point as it would run long enough for reshock to be captured, but would stop the simulation before expansion waves reflected from the upstream boundary could intersect the interface. The viscosity model was not included in these resolution study simulations because the viscosity study and consequent decision to include viscosity was dependent on the resolution study.

All but two of these simulations were stopped at less than $0.1 \mathrm{~ms}$ simulation time. Two simulations, at $282 \mu \mathrm{m}$ and $56 \mu \mathrm{m}$ resolutions, were run out to a time of $0.5 \mathrm{~ms}$ where the interface had developed long enough for a qualitative comparison to be made. Density plots of these two simulations are shown below in figure 13. It can be seen from these plots that the $56 \mu \mathrm{m}$ resolution case showed fine structures that one would expect to be damped out by species diffusion. Since species diffusion could not be included in the simulations the $282 \mu \mathrm{m}$ resolution simulation provides a more density plot that is expected to match experiments more closely. Computational times for these simulations were significantly different as well. These simulations were run on 128 cores total, where each node consisted of AMD Optetron quad core processors with 16 cores and 32GB of memory per node. The $56 \mu \mathrm{m}$ resolution case required and estimated $130 \mathrm{hrs}$ to run, and the $282 \mu \mathrm{m}$ case required $7 \mathrm{hrs}$. The next incremental increase in resolution $(169 \mu \mathrm{m})$ gave an estimated run time of $32 \mathrm{hrs}$. Based on the computational times the $282 \mu \mathrm{m}$ resolution was selected for the parametric study.
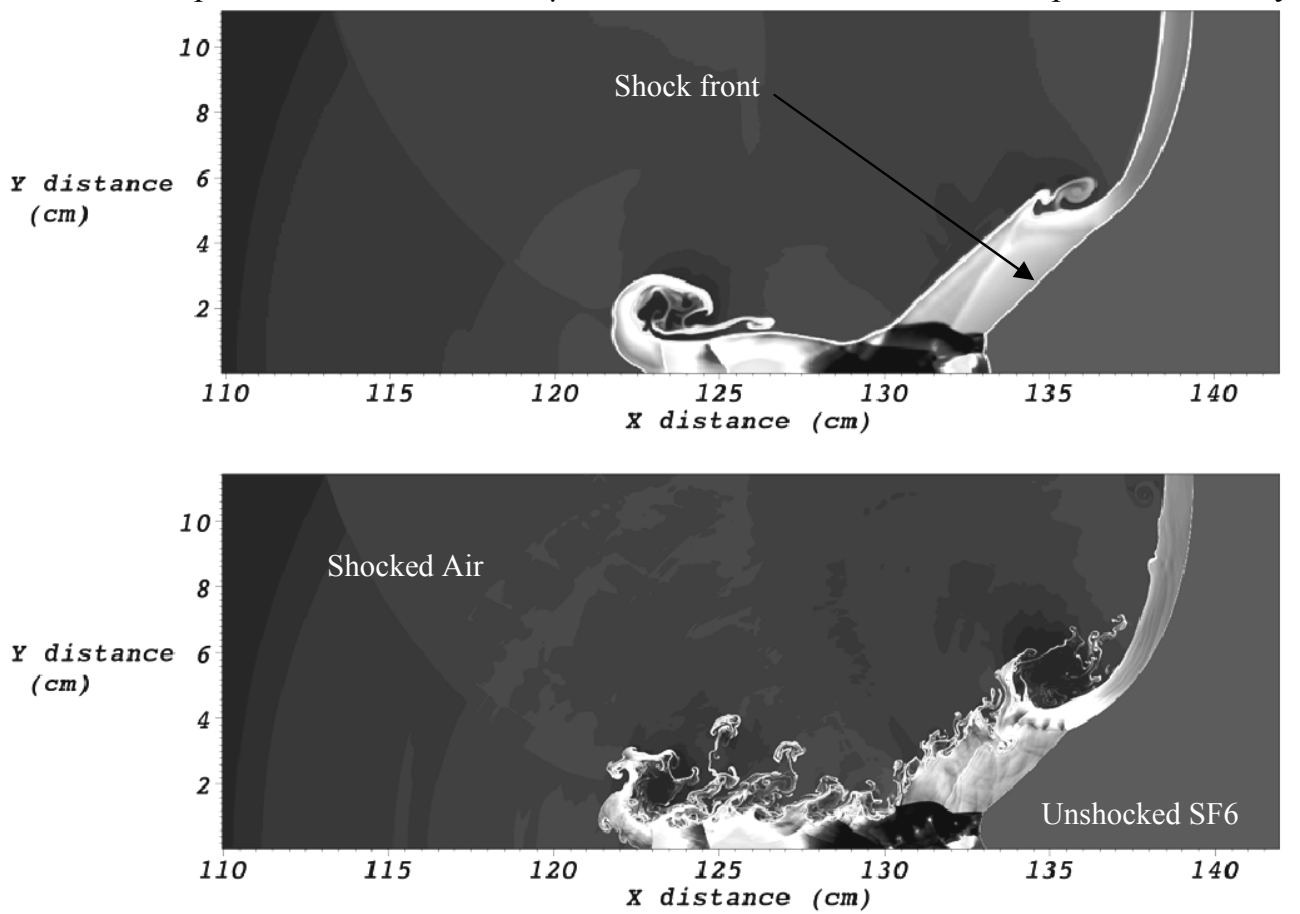

FIG. 13: Density plot for $\mathrm{M}_{\mathrm{i}}=2.5, \mathrm{~A}_{\text {int }}=30^{\circ}$, and At $\sim .67$ (air-SF6 gas pair) at $\mathrm{t}=0.5 \mathrm{~ms}$. Top: resolution of $282 \mu \mathrm{m}$. Bottom: resolution of $56 \mu \mathrm{m}$. 


\section{APPENDIX B: VISCOSITY STUDY}

After selecting the resolution for the parametric study, a comparison was made between a viscous simulation and an inviscid simulation. The same parameters used in the resolution study were again applied, incident shock Mach number of 2.5, inclination angle of 30 degrees, and air-SF6 gas pair. Each simulation was run to greater than $4 \mathrm{~ms}$ to allow late time and reshock development to be compared. Figure 14 below shows density plots for the viscous and inviscid cases at approximately $1 \mathrm{~ms}$. From these plots it can be seen that the addition of viscosity has a significant damping effect on the growth of secondary spikes. The addition of viscosity has also slowed the transmitted wall reflected shockwave due to the presence of a boundary layer and stretched the interface due to the no slip condition at the wall. At later times the difference becomes more significant but more difficult to define. The viscous model was chosen because of the significant differences in the interface growth. Including viscosity in the simulation increased the computational time from 7 hours to 11 hours for the parameters $\mathrm{M}=2.5, \mathrm{~A}=30^{\circ}$, air-SF6.
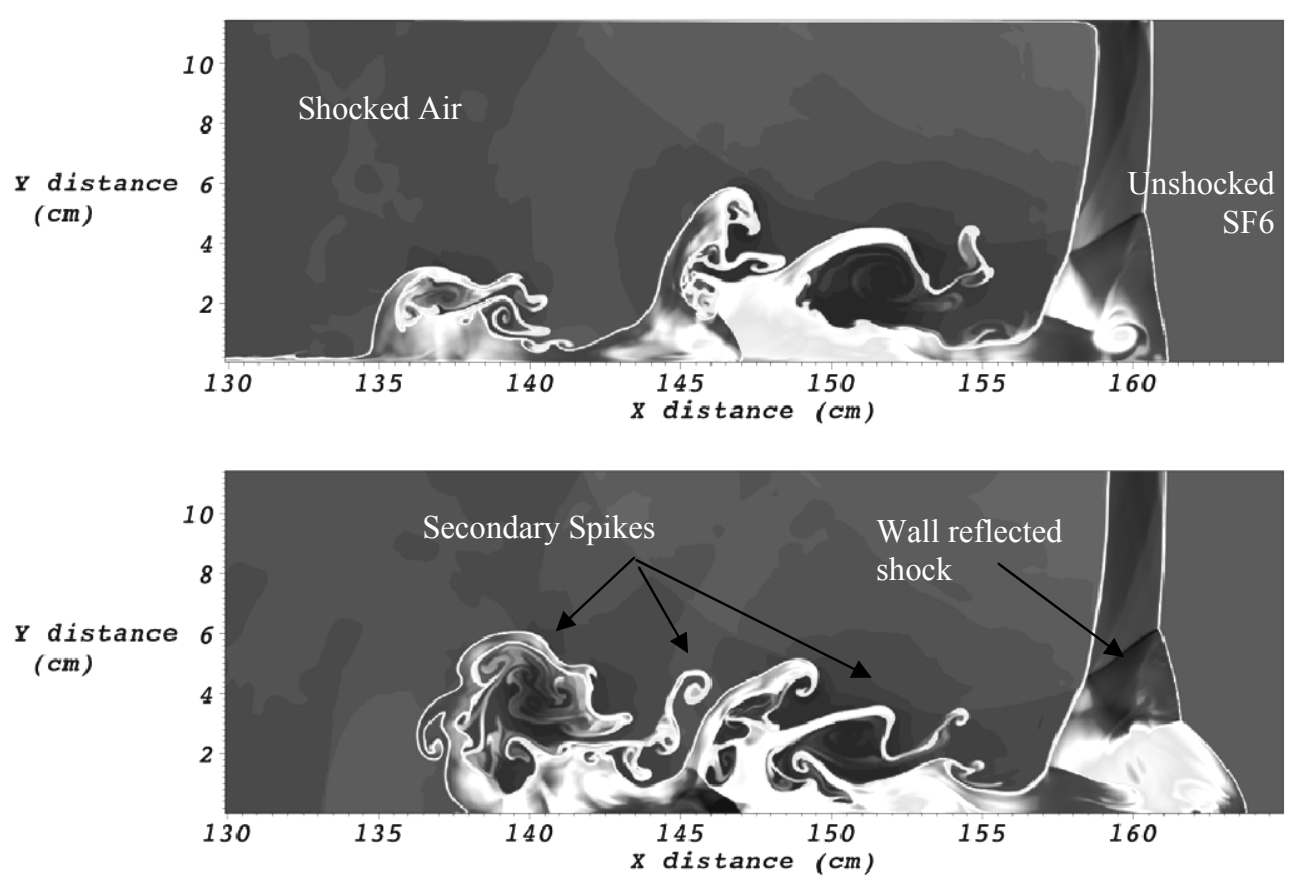

FIG. 14: Density plots for $M_{i}=2.5, A_{\text {int }}=30^{\circ}$, and $A t \sim 0.67$ (air-SF6 gas pair) at $t=1 \mathrm{~ms}$. Top: viscous model. Bottom: inviscid model. 


\section{REFERENCES}

[1] R. D. Richtmyer, Comm. Pure Appl. Math. 13, 297-319 (1960).

[2] E. E. Meshkov, Fluid Dyn 4, 101-104 (1972).

[3] M. Brouillette, Annu. Rev. Fluid Mech 34, 445-468 (2002).

[4] N. J. Zabusky, Annu. Rev. Fluid Mech 31, 495-536 (1999).

[5] V. V. Krivets, C. C. Long, J. W. Jacobs, and J. A. Greenough, Shock Waves 1205-1210 (2009).

[6] B. Motl, J. Oakley, D. Ranjan, C. Weber, M. Anderson, and R. Bonazza, Phys. Fluids 21, 126102 (2009).

[7] D. Ranjan, M. Anderson, J. Oakley, and R. Bonazza, Phys. Rev. Lett. 94, 184507 (2005); D. Ranjan, J. Niederhaus, B. Motl, M. Anderson, J. Oakley, and R. Bonazza, Phys. Rev. Lett. 98, 024502 (2007); D. Ranjan, J. Oakley and R. Bonazza, Annu. Rev. Fluid Mech., 43: 117-40 (2011).

[8] K. Prestridge, P. Vorobieff, P. M. Rightley, and R. F. Benjamin, Phys. Rev. Lett. 84, 4353-4356 (2000).

[9] P. R. Chapman and J. W. Jacobs, Phys. Fluids 18, 074101 (2006).

[10] M. L. Wilkins, Calculation of Elastic Plastic Flow (University of California Lawrence Radiation Labratory, 1963).

[11] T. V. Kolev and R. N. Rieben, Journal of Computational Physics 228, 8336-8366 (2009).

[12] R. W. Sharp and R. T. Barton, HEMP Advection Model (Lawrence Livermore Labratory, 1981).

[13] M. J. Berger and J. Oliger, J. Comput. Phys. 53, 484-512 (1984).

[14] M. J. Berger and P. Colella, J. Comput. Phys. 82, 64-84 (1989).

[15] K. H. Winkler, J. W. Chalmers, S. W. Hodson, P. R. Woodward, and N. J. Zabusky, Phys. Today 29 (1987).

[16] G. Taylor, Proc. Royal Soc. London. A, Mathematical and Physical Sciences 192-196 (1950).

[17] K. A. Meyer and P. J. Blewett, Phys. Fluids 15, 753 (1972).

[18] M. Vandenboomgaerde, C. Mü̈gler, and S. Gauthier, Phys. Rev. E 58, 1874-1882 (1998).

[19] R. Samtaney and N. J. Zabusky, J. Fluid Mech. 269, 45-78 (2006).

[20] S. Zhang, G. Peng, and N. J. Zabusky, J. Turbul. 6, (2005).

[21] K. O. Mikaelian, Phys. Fluids 17, 034101 (2005).

[22] W. H. Cabot and A. W. Cook, Nat. Phys. 2, 562-568 (2006). 